\title{
Diagnostic performance of microRNA-133a in acute myocardial infarction: A meta-analysis
}

\author{
Lei Zhu, Fuyuan Liu, Hua Xie, Jin Feng
}

Department of Cardiology, Xiangyang Hospital Affiliated of Hubei Medical College, China

\begin{abstract}
Background: The aim of this study was to evaluate the diagnostic performance of microRNA-133a in the diagnosis of acute myocardial infarction (AMI).

Methods: Major databases including PubMed, Embase and the Cochrane Library were searched for case-controlled studies comparing AMI and non-AMI patients. The outcome was evaluated by the relative expression of microRNA-133a in plasma or serum. The Mantel-Haenszel odds ratio (OR) was calculated using a fixed-effects model meta-analysis for the outcome. The primary outcomes of interest were pooled sensitivity, specificity and diagnostic accuracy of microRNA-133a for AMI.

Results: Out of 137 identified related articles, 10 were found to conform with the inclusion and exclusion criteria of the study. The 10 case-controlled studies contained complete data for 1,074 patients (with no restrictions of race, age or sex), and a database containing 137 patients from the registry of each study. In addition to low heterogeneity, a statistically significant increase was found in overall microRNA-133a expression between AMI vs. non-AMI; the pooled OR was 22.84 (95\% confidence interval [CI] 13.87-37.63), sensitivity was 0.84 (95\% CI 0.75-0.90), specificity was 0.82 (95\% CI 0.74-0.89) and area under curve (AUC) was 0.90 (95\% CI 0.87-0.92).

Conclusions: Based on the meta-analysis of ten case-controlled studies including 1,074 patients, it was found that the level of microRNA-133a in blood serum or plasma maybe used as a diagnostic biomarker of AMI. (Cardiol J 2018; 25, 2: 260-267)
\end{abstract}

Key words: diagnostic accuracy/value/performance, acute myocardial infarction, microRNA-133a/miR-133a, meta-analysis

\section{Introduction}

Myocardial injury resulting from acute myocardial infarction (AMI) can cause blood circulation disorder and chest discomfort similar to heartburn symptoms, and can even lead to heart failure or cessation of blood flow [1]. AMI is a common coronary artery disease, with risk factors including smoking and hypertension present in nearly $90 \%$ of patients [2]. Environmental pollution, including noise and air pollution, can also have adverse effects on myocardial infarction (MI) [3]. The pathogenesis of AMI involves the accumulation of collagen fibers, resulting in myocardial fibrosis. Usually, healthy people have low expression levels of collagen, but in patients with MI the affected tissue and surrounding area have an increased expression [4]. Although diagnostic, assessment and therapeutic techniques such as ultrasound, biomarkers such as the C3G protein and stem cell transplantation, respectively, have been used successfully in detection, diagnosis and treatment of myocardial injury [5-7], there is still a need to further explore novel approaches for the diagnosis and treatment of AMI and to understand the pathophysiology of AMI.

MicroRNA (miRNA) is a short chain geneediting, noncoding single-stranded ribonucleotide that can bind to messenger RNA (mRNA) on its poly-adenylated tail end (3'-UTR) in the noncoding region to inhibit or promote its degradation

Address for correspondence: Lei Zhu, MD, Xiangyang Hospital, Hubei Medical College, 441000, China, tel: +86-15072375180, e-mail: zhuleill137@163.com

Received: 26.07.2017

Accepted: 07.09.2017 
[8]. One kind of miRNA can interact with a variety of mRNAs, and one mRNA may interact with a variety of miRNAs together forming a sophisticated regulatory system. Recently, many studies have shown that after MI, the detection of blood miRNA expression can be used as a diagnostic and prognostic tool for AMI. For example, miRNA-21 expression can be increased to reduce the size of the infarct injury [9], miRNA-1 can be used as a potential molecular biomarker for AMI [10], and miRNA-133a can be used as a long-term prognostic indicator for patients after MI [11].

Studies have shown that the miRNA-133a expression level in blood and is related to AMI [12], and this interplay suggests that miRNA-133a could be used as a diagnostic molecular biomarker of AMI [13, 14]. MicroRNA-133a is a member of the miRNA-133 family. The aim of the present study is to determine the diagnostic value of the miRNA-133a expression level for AMI using a statistical method of meta-analysis.

\section{Methods}

\section{Document retrieval}

Keywords were searched in PubMed, Medline and Embase databases using the following medical subject headings (MeSH): "microRNA-133a", "miR-133a", and "myocardial infarction" The data was obtained by retrieving relevant literature references based on information about the studies included.

\section{Inclusion and exclusion criteria}

Two reviewers independently screened all related titles and abstracts using the following inclusion and exclusion criteria. Inclusion criteria: 1) patients with clinical diagnosis of AMI; 2) the published study was a case-control design study; 3) the study was originally published in English within the past 10 years (range from 2006.11 to 2016.11$)$; 4) the study provides accurate and complete information about AMI patients; 5 ) the study was assessed as a high-quality study; 6 ) the target miRNA of samples was normalized to an endogenous miRNA with the calculation formula $2^{-\Delta \Delta \mathrm{Ct}}$ : 2exp (mean Ct endogenous controls $-\mathrm{Ct}$ target miRNA). Exclusion criteria: 1) patients with congenital heart disease; 2 ) a review of the literature, meetings and correspondence letters; 3) experimental design involved an animal model; 4) specimen source from tissue, secretions or excretions.
Evaluation of the quality of included studies

Two investigators systematically assessed the quality of each article included in the meta-analysis according to the Quality Assessment of Diagnostic Accuracy Studies tool (QUADAS-2) with 11 entries for the answer "yes", "no" or "unclear". The "yes" answer is given a plus one-point score, the "no" answer is given a minus one-point score, and the "unclear" answer contributed to zero-point. A score of 7 points or more was considered high quality. Discrepancies were resolved by discussion to reach a consensus.

\section{Data extraction}

Two reviewers independently extracted relevant data required for research purposes from the studies included. When discrepancies were encountered, either they were discussed until differences were settled or the two reviewers were assisted by a third staff member until a consensus was reached. Data extracted from the literature included: first author, year of publication, source documents, case or control group of patients who participated in the study, the method of detection of miRNA and type of specimen.

\section{Statistical analysis}

After successful extraction of data, Stata 14.0 software was used for the meta-analysis. The pooled odds ratios (OR) were calculated and the associated 95\% confidential intervals (CIs) using a fixed or random effects model with the statistical method of Mantel-Haenszel (M-H) or DerSimonian-Laird (D-L). Thevariability issue was addressed in results across studies by using the $\mathrm{I}^{2}$ statistic and $\mathrm{p}$-value obtained following the meta-analysis instructions of Stata. The analysis results include 1) publication bias and heterogeneity of included studies; 2) combined effect of the size of included studies; 3 ) being included in the study sensitivity, specificity, and diagnostic OR; and 4) subgroup analysis based on type of AMI: ST-segment elevation myocardial infarction (STEMI) and non-ST-segment elevation myocardial infarction (NSTEMI).

Publication bias analysis was conducted with a comprehensive program of statistical and graphical routines for undertaking meta-analysis of diagnostic performance in Stata. Primary data combination is performed within the bivariate mixed-effects binary regression modeling framework. The model specification, estimation and prediction are carried out following the instructions in Stata release 14.0 (StataCorp LP, College Station, TX, USA). 


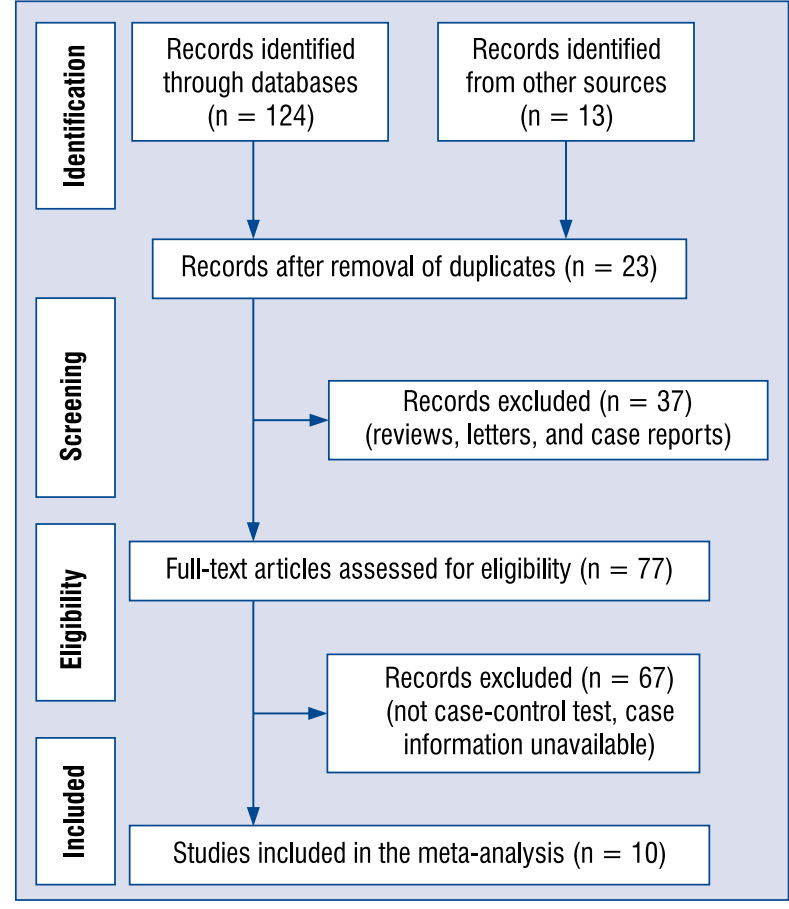

Figure 1. Flowchart of the study selection process.

Using coefficients and variance-covariance matrices estimated by the optimal model, the summary operating sensitivity and specificity (with confidence and prediction contours in the summary receiver operating characteristic [ROC] curve space), the summary likelihood and ORs were also calculated. The global and relevant test performance metric-specific heterogeneity statistics are also provided. Studies of the meta-analytical integration of the diagnostic accuracy facilitate the extensive statistical and graphical data synthesis and exploratory analyses of the heterogeneity, covariate effects, publication bias and impact. The Bayes' nomograms and likelihood ratio matrices may be obtained and used to guide the clinical decision-making process.

\section{Results}

\section{Literature search results}

The initial literature search retrieval process is shown in Figure 1 total of 137 publications relevant to miRNA-133a in the English literature were searched, according to the inclusion and exclusion criteria. In summation, 10 articles met the inclusion criteria [12-20]. The documents included are contained in the basic information as shown in Tables 1 and 2.

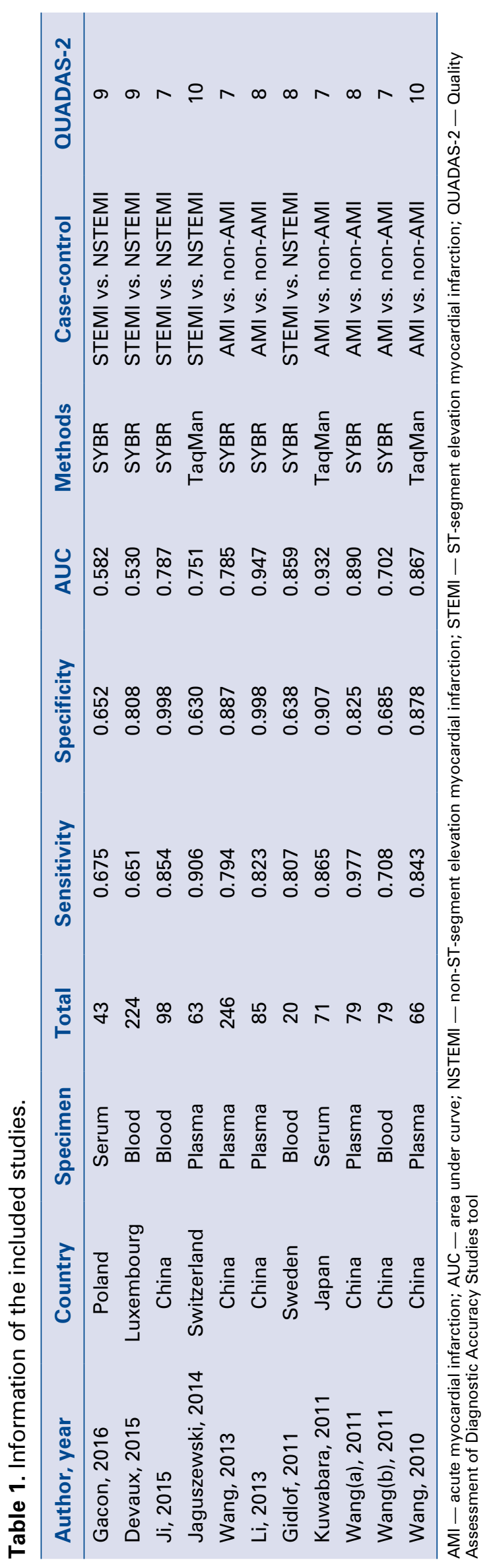




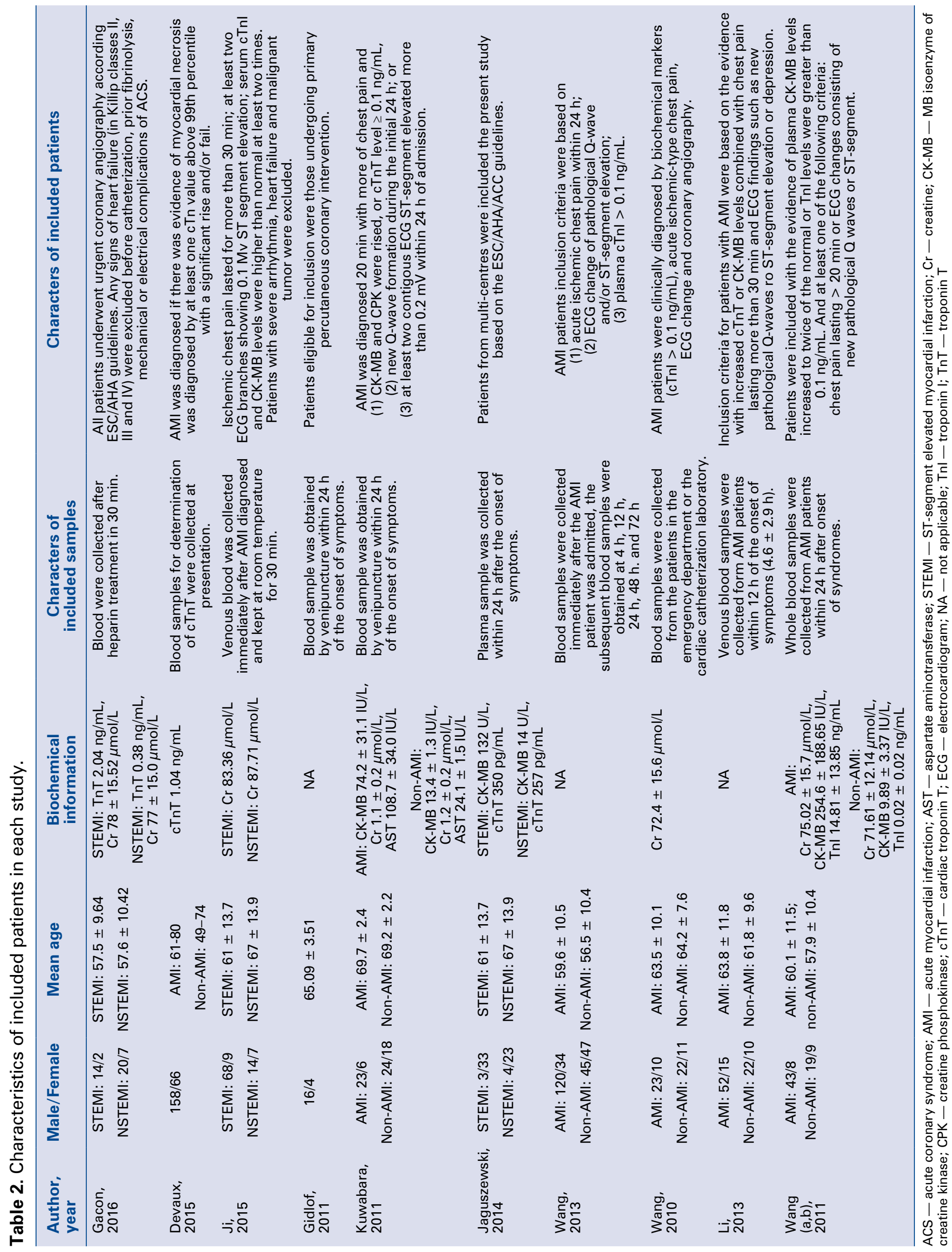




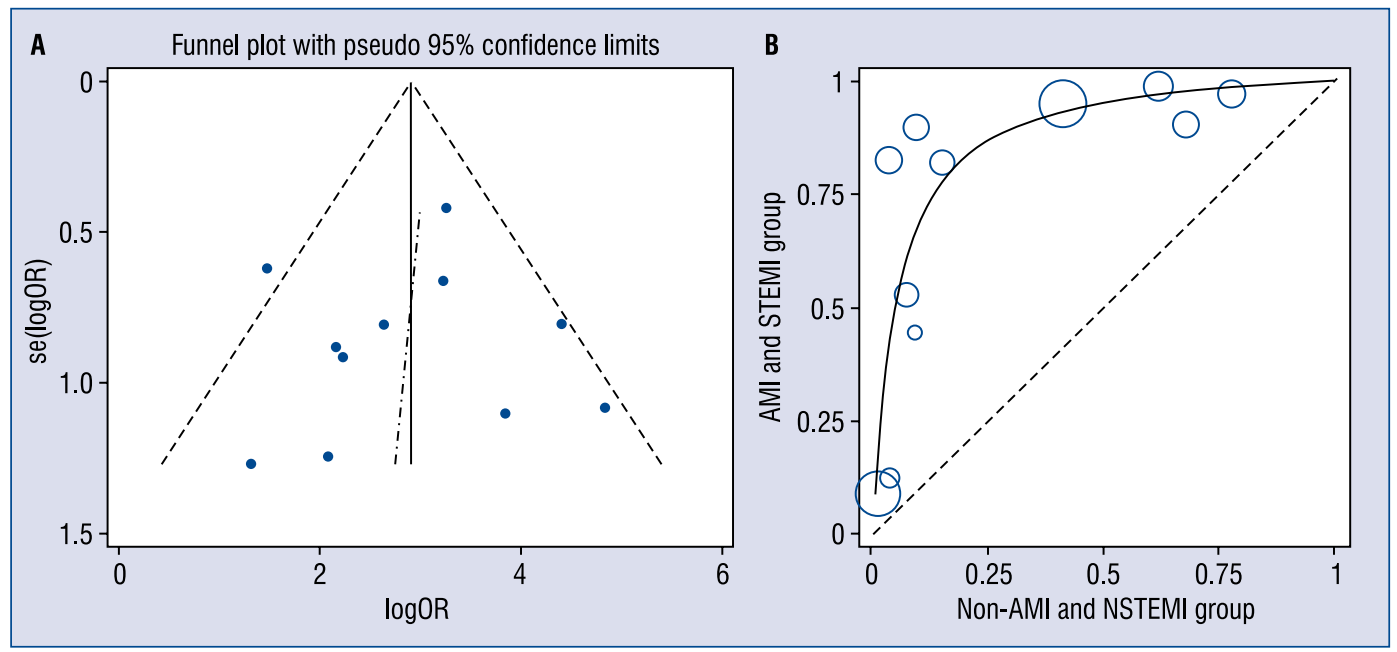

Figure 2. Publication bias test and heterogeneity of the included studies. A. Funnel plot of the included studies; B. Labbe plot for detecting heterogeneity; AMI - acute coronary syndrome; NSTEMI — non-ST-segment elevation myocardial infarction; OR - odds ratio.

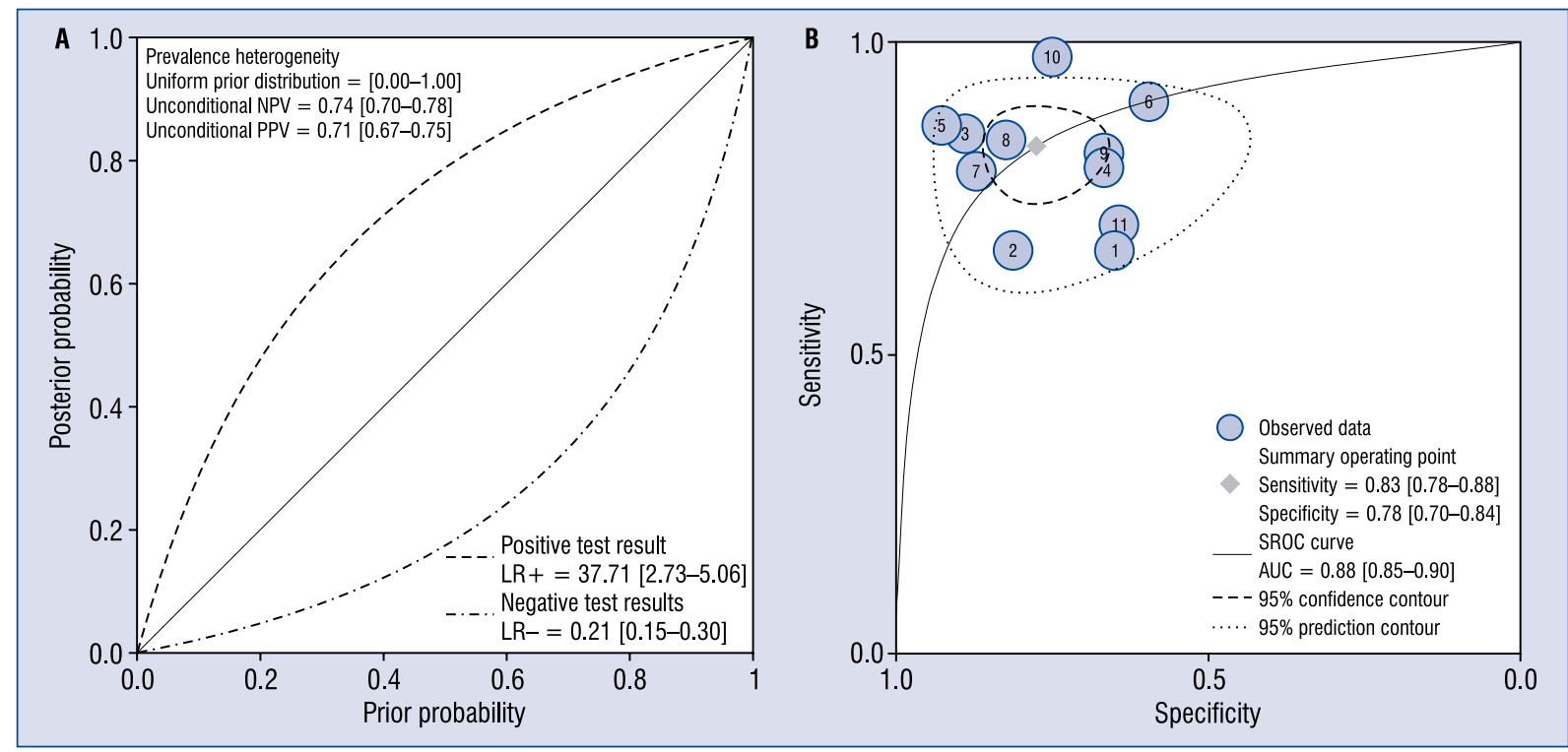

Figure 3. Diagnostic probabilities and summary receiver operating characteristic (SROC) curve; A. Line graph of post-test probabilities versus prior probabilities between 0 and 1 using summary likelihood ratios; $\mathrm{Cl}$ - confidence interval; NPV - negative predictive value; PPV - positive predictive value; B. Summary receiver operating characteristic curve; AUC - area under curve.

\section{Document publication bias and heterogeneity test}

The results of Begg's rank correlation test were as follows $z=-0.7, p=0.484$; Egger regression analysis $\mathrm{t}=-0.23, \mathrm{p}=0.824$; and the effect of size in detecting heterogeneity was $\mathrm{I}^{2}=41.7 \%$, $\mathrm{p}=0.071$. The results showed that studies included have moderate publication bias (Fig. 2A), and low heterogeneity $\left(25 \%<\mathrm{I}^{2}<50 \%\right.$; Fig. 2B). Accordingly, a fixed effect model to merge the effect size of the studies included was selected.

\section{Meta-analysis and subgroup meta-analysis}

The combined analysis of the included studies revealed that sensitivity was 0.83 (95\% CI 0.78 $-0.88)$, the specificity was 0.78 (95\% CI $0.70-0.84)$, 


\begin{tabular}{|c|c|c|c|}
\hline Author, year & & Odds ratio (95\% Cl) & $\%$ Weight \\
\hline Wang GK, 2010 & $-\frac{1}{p}-$ & $25.20(6.87-92.40)$ & 12.97 \\
\hline Kuwabara Y, 2011 & & $82.33(16.99-398.88)$ & 4.82 \\
\hline Wang R(a), 2011 & & $126.00(15.10-1051.61)$ & 3.25 \\
\hline Wang R(b), 2011 & $-\cdot-$ & $4.36(1.29-14.72)$ & 34.31 \\
\hline Wang F, 2013 & $\begin{array}{r}1 \\
-4 \\
-4\end{array}$ & $25.93(11.38-59.11)$ & 35.26 \\
\hline Li YQ, 2013 & $\longrightarrow-1$ & $9.29(1.55-55.78)$ & 9.40 \\
\hline Overall $\left(l^{2}=62.2 \%, p=0.021\right)$ & \rangle & $22.84(13.87-37.63)$ & 100.00 \\
\hline 0.00095 & \multicolumn{3}{|c|}{1052} \\
\hline
\end{tabular}

Figure 4. Forest plot comparing acute myocardial infarction (AMI) and non-AMI in relation to expression of miR-133a; $\mathrm{Cl}$ - confidence interval.

\begin{tabular}{|c|c|c|c|}
\hline Author, year & & Odds ratio (95\% Cl) & $\%$ Weight \\
\hline Gidlof 0, 2011 & & $8.00(0.70-91.80)$ & 15.49 \\
\hline Jaguszewski M, 2014 & & $13.97(2.87-67.96)$ & 33.44 \\
\hline Devaux Y, 2015 & & $8.63(1.53-48.75)$ & 22.68 \\
\hline Ji Q, 2015 & & $46.77(5.39-405.74)$ & 8.22 \\
\hline Gacon J, 2016 & $一-$ & $3.71(0.31-44.66)$ & 20.17 \\
\hline Overall $\left(I^{2}=0.0 \%, p=0.615\right)$ & & $12.46(5.24-29.63)$ & 100.00 \\
\hline 0.00246 & 1 & 106 & \\
\hline
\end{tabular}

Figure 5. Forest plot comparing ST-segment elevation myocardial infarction (STEMI) and non-NSTEMI in relation to expression of miR-133a; $\mathrm{Cl}$ - confidence interval.

positive likelihood ratio was 3.7 (95\% CI 2.7-5.1), the negative likelihood ratio was 0.21 (95\% CI $0.15-0.30)$, the diagnostic OR was $17(95 \% \mathrm{CI}$ 10-30), the area under the summary ROC curve was 0.88 (95\% CI 0.85-0.90) (Fig. 3A, B).

Additionally, a meta-regression analysis was performed to determine the reason for the moderate heterogeneity. Results showed that moderate heterogeneity originated from the difference between the AMI and STEMI and the different methods of detecting miRNA-133a. Thus, a subgroup meta-analysis was performed based on results of the meta-regression analysis. Combined results of AMI vs. non-AMI are shown in the figures as follows (Fig. 4), pooled OR was 22.84 (95\% CI 13.87-37.63), $\mathrm{I}^{2}=62.2 \%, \mathrm{p}=0.021$; STEMI vs. NSTEMI; (Fig. 5), pooled OR was 12.46 (95\% CI 5.24-29.63), $\mathrm{I}^{2}=0.0 \%, \mathrm{p}=0.615$. The pooled OR of the TaqMan detection method was 28.29 (95\% CI 12.00-66.68), $\mathrm{I}^{2}=21.7 \%, \mathrm{p}=0.279$; The pooled OR of the SYBR detection method was 17.01 (95\% CI 10.30-28.09), $\mathrm{I}^{2}=45.8 \%$, $\mathrm{p}=0.074$ (Fig. 6).

\section{Discussion}

This analysis showed that the expression level of miRNA-133a after AMI is increased in plasma and serum, and confirmed the diagnostic performance of miRNA-133a during the formation of AMI. This finding has important clinical significance for early diagnosis of AMI and its treatment. MicroRNAs are a class of endogenous short RNA fragments, that do not encode proteins, but are involved in many biological processes associated with signal transduction, and therefore they have been used to diagnose heart failure or hypertensive patients [21, 22]. In addition, some reports confirmed that miRNAs could perhaps be used as molecular biomarkers for diagnosis and prognosis of MI diagnosis and prognosis [23, 24]. Using a number of case-control studies, a comprehensive analysis was performed of the expression of miRNA-133a in patients with AMI and non-AMI in their blood, and results showed increased expression of miRNA-133a in blood of patients with AMI. Moreover, results of a summary ROC curve 


\begin{tabular}{|c|c|c|c|}
\hline \multicolumn{2}{|l|}{ Author, year } & \multirow[t]{2}{*}{ Odds ratio $(95 \% \mathrm{Cl})$} & \multirow[t]{2}{*}{$\%$ Weight } \\
\hline SYBR & & & \\
\hline Gidlof 0, 2011 & $-\infty$ & $8.00(0.70-91.80)$ & 4.88 \\
\hline Wang R(a), 2011 & & $-126.00(15.10-1051.61)$ & 2.23 \\
\hline Wang $R(b), 2011$ & $-\cdot-$ & $4.36(1.29-14.72)$ & 23.49 \\
\hline Wang F, 2013 & & $25.93(11.38-59.11)$ & 24.14 \\
\hline Li YQ, 2013 & $\longrightarrow-$ & $9.29(1.55-55.78)$ & 6.43 \\
\hline Devaux Y, 2015 & $\longrightarrow-$ & $8.63(1.53-48.75)$ & 7.15 \\
\hline Ji Q, 2015 & & $46.77(5.39-405.74)$ & 2.59 \\
\hline Gacon J, 2016 & 一⿻上丨- & $3.71(0.31-44.66)$ & 6.36 \\
\hline Subtotal $\left(l^{2}=45.8 \%, p=0.074\right.$ & & $17.01(10.30-28.09)$ & 77.28 \\
\hline TaqMan & & & \\
\hline Wang GK, 2010 & - & $25.20(6.87-92.40)$ & 8.88 \\
\hline Kuwabara Y, 2011 & & $82.33(16.99-398.88)$ & 3.30 \\
\hline Jaguszewski M, 2014 & 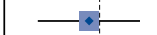 & $13.97(2.87-67.96)$ & 10.54 \\
\hline Subtotal $\left(l^{2}=21.7 \%, p=0.279\right.$ & & $28.29(12.00-66.68)$ & 22.72 \\
\hline Overall $\left(l^{2}=41.7 \%, p=0.071\right.$ & $\wedge$ & $19.57(12.71-30.14)$ & 100.00 \\
\hline 0.00095 & 1 & 1052 & \\
\hline
\end{tabular}

Figure 6. Forest plot of different detection methods of the expression of miR-133a; $\mathrm{Cl}$ - confidence interval.

analysis suggest that miRNA-133a may be used for diagnosis of AMI patients.

Additionally, an interesting idea was sparked by the subgroup analysis result: the TaqMan detection method for miRNA-133a may be more accurate than the SYBR Green detection method. Based on TaqMan had pooled OR value 28.29 vs. 17.01 of the SYBR Green, calculations for each group's AUC was done, also an un-paired test between the two groups ORs was performed. The results are positive and statistically significant according to these findings. That is to say, the TaqMan detection method is superior to the SYBR Green method just as the other authors have described in prior studies [25].

Numerous studies have investigated whether miRNA-133a could be an optimal biomarker for patients with AMI. The results showed inconsistency in the diagnostic value of miRNA-133a. The results of several study analyses support a role for miRNA-133a as a biomarker [16, 19, 23, 24, $26]$. However, the number of AMI patients and healthy controls participating in these studies were relatively small. Widera et al. [27] reported that there was a large overlap between patients with unstable angina or $\mathrm{MI}$ in relation to the level of miRNA-133a. Kuwabara et al. [12] reported significantly increased serum levels of miRNA-133a in patients with AMI, as well as in patients with unstable angina pectoris. These studies had a larger sample size than the studies mentioned above and support a role for miRNA-133a as a diagnostic biomarker. Additionally, the present data adequately supports the suggestion that miRNA-133a could be used as a biomarker for
AMI diagnosis, particularly considering that the pooled AUC of the ROC curve is 0.88 (95\% CI 0.85-0.90) (Fig. 3B). Overall, the results of this meta-analysis indicate that miRNA-133a can be used as a diagnostic biomarker for AMI.

Recently, many studies have shown that miRNA circulating in the blood may affect mortality in patients with AMI [11, 27], and miRNA concentration may influence the prognosis of patient 1-year survival $[28,29]$. In addition, a few studies have shown that miRNAs are involved in myocardial remodeling [30, 31]. Although, according to the results of these reports, the molecular mechanism of miRNA involvement in AMI remains largely unclear, the miRNA may still be used as molecular diagnosis biomarker of AMI. Moreover, the results of the hierarchical summary ROC curve analysis were also consistent with other results of this meta-analysis.

\section{Limitations of the study}

This study has its own limitations. First, analysis is based on a relatively small study population. Additionally, there are individual and regional differences between research groups. Moreover, a larger group of patients should be included in this study to support more reliable results. In addition, the studies included miRNA expression levels were measured only at one point in time, with no continuous detection. which reduces the reliability of this study.

\section{Conclusions}

In short, the present study confirmed the diagnostic role of miRNA-133a in AMI patients. 
However, compared with the present gold standard biomarkers such as creatine troponin T, troponin I and MB isoenzyme of creatine kinase, miRNA is not the best candidate at the present time due to protocol and cost of these detection methods. So, before miRNA-133a becomes commonly used for AMI patients clinically. Further investigation should be conducted to obtain accurate statistical analyses, and results should be subjected to further inquiry and analysis.

\section{Conflict of interest: None declared}

\section{References}

1. Zhou C, Cui Q, Su G, et al. MicroRNA-208b Alleviates PostInfarction Myocardial Fibrosis in a Rat Model by Inhibiting GATA4. Med Sci Monit. 2016; 22: 1808-1816, indexed in Pubmed: 27236543.

2. White HD, Chew DP. Acute myocardial infarction. Lancet. 2008; 372(9638): 570-584, doi: 10.1016/S0140-6736(08)61237-4, indexed in Pubmed:18707987.

3. Huss A, Spoerri A, Egger M, et al. Aircraft Noise, Air Pollution, and Mortality From Myocardial Infarction. Epidemiology. 2010; 21(6): 829-836, doi:10.1097/ede.0b013e3181f4e634.

4. Cleutjens JP, Verluyten MJ, Smiths JF, et al. Collagen remodeling after myocardial infarction in the rat heart. Am J Pathol. 1995; 147(2): 325-338, indexed in Pubmed: 7639329.

5. Sun J, Rong Z, Wugeti N, et al. Experimental evaluation of myocardial fibrosis in a rapid atrial pacing model in New Zealand rabbits using quantitative analysis of ultrasonic backscatter. Med Sci Monit. 2014; 20: 1884-1889, doi: 10.12659/MSM.891242, indexed in Pubmed: 25296565.

6. Yang S, Piao J, Jin L, et al. Does pretreatment of bone marrow mesenchymal stem cells with 5-azacytidine or double intravenous infusion improve their therapeutic potential for dilated cardiomyopathy? Med Sci Monit Basic Res. 2013; 19: 20-31, indexed in Pubmed: 23314418.

7. Wang L, Li G, Wang Z, et al. Elevated expression of C3G protein in the peri-infarct myocardium of rats. Med Sci Monit Basic Res. 2013; 19: 1-5, indexed in Pubmed: 23291598.

8. Bartel DP. MicroRNAs: genomics, biogenesis, mechanism, and function. Cell. 2004; 116(2): 281-297, indexed in Pubmed: 14744438

9. Dong S, Cheng Y, Yang J, et al. MicroRNA expression signature and the role of microRNA-21 in the early phase of acute myocardial infarction. J Biol Chem. 2009; 284(43): 29514-29525, doi: 10.1074/jbc.M109.027896, indexed in Pubmed: 19706597.

10. Ai J, Zhang R, Li Y, et al. Circulating microRNA-1 as a potential novel biomarker for acute myocardial infarction. Biochem Biophys Res Commun. 2010; 391(1): 73-77, doi: 10.1016/j. bbrc.2009.11.005, indexed in Pubmed: 19896465.

11. Gidlöf O, Smith JG, Miyazu K, et al. Circulating cardio-enriched microRNAs are associated with long-term prognosis following myocardial infarction. BMC Cardiovasc Disord. 2013; 13: 12, doi: 10.1186/1471-2261-13-12, indexed in Pubmed: 23448306.

12. Kuwabara Y, Ono K, Horie T, et al. Increased microRNA-1 and microRNA-133a levels in serum of patients with cardiovascular disease indicate myocardial damage. Circ Cardiovasc Genet. 2011; 4(4): 446-454, doi: 10.1161/CIRCGENETICS.110.958975, indexed in Pubmed: 21642241.

13. Ke-Gang J, Zhi-Wei Li, Xin Z, et al. Evaluating diagnostic and prognostic value of plasma miRNA133a in acute chest pain patients undergoing coronary angiography. Medicine (Baltimore). 2016; 95(17): e3412, doi: 10.1097/MD.0000000000003412, indexed in Pubmed: 27124025.

14. Gacoń J, Kabłak-Ziembicka A, Stępień E, et al. Decision-making microRNAs (miR-124, -133a/b, -34a and -134) in patients with occluded target vessel in acute coronary syndrome. Kardiol Pol.
2016; 74(3): 280-288, doi: 10.5603/KP.a2015.0174, indexed in Pubmed: 26365938.

15. Devaux Y, Mueller M, Haaf P, et al. Diagnostic and prognostic value of circulating microRNAs in patients with acute chest pain. J Intern Med. 2015; 277(2): 260-271, doi: 10.1111/joim.12183, indexed in Pubmed: 24345063.

16. Ji Q, Jiang Q, Yan W, et al. Expression of circulating microRNAs in patients with ST segment elevation acute myocardial infarction. Minerva Cardioangiol. 2015; 63(5): 397-402, indexed in Pubmed: 26198874.

17. Jaguszewski M, Osipova J, Ghadri JR, et al. A signature of circulating microRNAs differentiates takotsubo cardiomyopathy from acute myocardial infarction. Eur Heart J. 2014; 35(15): 999-1006, doi: 10.1093/eurheartj/eht392, indexed in Pubmed: 24046434.

18. Gidlöf O, Andersson P, van der Pals J, et al. Cardiospecific microRNA plasma levels correlate with troponin and cardiac function in patients with ST elevation myocardial infarction, are selectively dependent on renal elimination, and can be detected in urine samples. Cardiology. 2011; 118(4): 217-226, doi: 10.1159/000328869, indexed in Pubmed: 21701171.

19. Wang R, Li N, Zhang Y, et al. Circulating microRNAs are promising novel biomarkers of acute myocardial infarction. Intern Med. 2011; 50(17): 1789-1795, indexed in Pubmed: 21881276.

20. Wang GK, Zhu JQ, Zhang JT, et al. Circulating microRNA: a novel potential biomarker for early diagnosis of acute myocardial infarction in humans. Eur Heart J. 2010; 31(6): 659-666, doi: 10.1093/eurheartj/ehq013, indexed in Pubmed: 20159880.

21. Goren Y, Meiri E, Hogan C, et al. Relation of reduced expression of MiR-150 in platelets to atrial fibrillation in patients with chronic systolic heart failure. Am J Cardiol. 2014; 113(6): 976-981, doi: 10.1016/j.amjcard.2013.11.060, indexed in Pubmed: 24462065 .

22. Schlosser K, White RJ, Stewart DJ. miR-26a linked to pulmonary hypertension by global assessment of circulating extracellular microRNAs. Am J Respir Crit Care Med. 2013; 188(12): 1472-1475, doi: 10.1164/rccm.201308-1403LE, indexed in Pubmed: 24328779 .

23. Peng L, Chun-guang Q, Bei-fang Li, et al. Clinical impact of circulating miR-133, miR-1291 and miR-663b in plasma of patients with acute myocardial infarction. Diagn Pathol. 2014; 9(1): 89, doi: 10.1186/1746-1596-9-89, indexed in Pubmed: 24885383.

24. Li YQ, Zhang MF, Wen HY, et al. Comparing the diagnostic values of circulating microRNAs and cardiac troponin $T$ in patients with acute myocardial infarction. Clinics (Sao Paulo). 2013; 68(1): 75-80, indexed in Pubmed: 23420161.

25. Cao H, Shockey JM. Comparison of TaqMan and SYBR Green qPCR methods for quantitative gene expression in tung tree tissues. J Agric Food Chem. 2012; 60(50): 12296-12303, doi: 10.1021/jf304690e, indexed in Pubmed: 23176309.

26. Wang F, Long G, Zhao C, et al. Plasma microRNA-133a is a new marker for both acute myocardial infarction and underlying coronary artery stenosis. J Transl Med. 2013; 11: 222, doi: 10.1186/1479-5876-11-222, indexed in Pubmed: 24053180.

27. Widera C, Gupta SK, Lorenzen JM, et al. Diagnostic and prognostic impact of six circulating microRNAs in acute coronary syndrome. J Mol Cell Cardiol. 2011; 51(5): 872-875, doi: 10.1016/j. yjmcc.2011.07.011, indexed in Pubmed: 21806992.

28. Matsumoto S, Sakata Y, Nakatani D, et al. A subset of circulating microRNAs are predictive for cardiac death after discharge for acute myocardial infarction. Biochem Biophys Res Commun. 2012; 427(2): 280-284, doi: 10.1016/j.bbrc.2012.09.039, indexed in Pubmed: 22995291.

29. Wang R, Li N, Zhang Y, et al. Circulating microRNAs are promising novel biomarkers of acute myocardial infarction. Intern Med. 2011; 50(17): 1789-1795, indexed in Pubmed: 21881276.

30. Devaux Y, Vausort M, McCann GP, et al. A panel of 4 microRNAs facilitates the prediction of left ventricular contractility after acute myocardial infarction. PLoS One. 2013; 8(8): e70644, doi: 10.1371/ journal.pone.0070644, indexed in Pubmed: 23967079.

31. Devaux Y, Vausort M, Azuaje F, et al. Low levels of vascular endothelial growth factor B predict left ventricular remodeling after acute myocardial infarction. J Card Fail. 2012; 18(4): 330-337, doi: 10.1016/j.cardfail.2012.01.010, indexed in Pubmed: 22464775 . 